\title{
NONDENSE ORBITS FOR ANOSOV DIFFEOMORPHISMS OF THE 2-TORUS
}

\author{
JIMMY TSENG
}

\begin{abstract}
Let $\lambda$ denote the probability Lebesgue measure on $\mathbb{T}^{2}$. For any $C^{2}$-Anosov diffeomorphism of the 2-torus preserving $\lambda$ with measure-theoretic entropy equal to topological entropy, we show that the set of points with nondense orbits is hyperplane absolute winning (HAW). This generalizes the result in [18, Theorem 1.4] for $C^{2}$-expanding maps of the circle.
\end{abstract}

\section{INTRODUCTION}

For a dynamical system $f: X \rightarrow X$ on a set $X$ with a topology, we say that a point has nondense forward orbit if its forward orbit closure is a proper subset of $X$, and we call the set of these points the nondense set and denote it by $N D(f)$. The study of nondense orbits is important for the interactions between ergodic theory and Diophantine approximation and, partly inspired by this, there has been much recent interest in these sets (see [1, 3, 15, 6, 15, 18, 20, 21] for example). Of particular interest is the size of $N D(f)$ and whether these sets have the winning property, which is a strengthening of having full Hausdorff dimension and is stable under intersections. (See Section 2 for the precise definition of the winning property.) Almost all of the known dynamical systems that have winning nondense sets are (piecewise) linear or come from automorphisms. A class of exceptions is the result by the author for $C^{2}$-expanding self-maps on the circle [18, Theorem 1.4]. There is also a recent result by $\mathrm{W}$. Wu [21, Section 2] for partially hyperbolic diffeomorphisms on compact manifolds in which the diffeomorphisms are conformal when restricted to the unstable manifolds or the unstable manifolds are one-dimensional. Wu's result, however, shows only that the nondense set when restricted to an unstable manifold is winning, but does not show that the whole nondense set is winning 11 General diffeomorphisms present a challenge in dimensions higher than one for determining whether the nondense set is winning or not.

1.1. Statement of results. The purpose of this brief note is to show that the members of a large, natural class of nonlinear self-maps on the 2-torus have winning nondense sets, thereby, giving a large, natural class of higher dimensional examples of winning nondense sets for more general diffeomorphisms. Since the winning property is stable under intersections, our results, in fact, also apply to nondense complete orbits. Let $\lambda$ denote the probability Lebesgue measure on $\mathbb{T}^{2}$. Our main result is the following.

TheOREM 1.1. Let $g: \mathbb{T}^{2} \rightarrow \mathbb{T}^{2}$ be a $C^{2}$-Anosov diffeomorphism preserving $\lambda$ such that $h_{\lambda}(g)=$ $h_{\mathrm{top}}(g)$. Let $\boldsymbol{x}_{0} \in \mathbb{T}^{2}$. Then the set

$$
N D\left(g, \boldsymbol{x}_{0}\right):=\left\{\boldsymbol{x} \in \mathbb{T}^{2}: \boldsymbol{x}_{0} \notin \overline{\left\{g^{n}(x)\right\}_{n=0}^{\infty}}\right\}
$$

is hyperplane absolute winning (HAW) and thus has full Hausdorff dimension.

The author acknowledges the research leading to these results has received funding from the European Research Council under the European Union's Seventh Framework Programme (FP/2007-2013) / ERC Grant Agreement n. 291147.

${ }^{1} \mathrm{Wu}$ 's result does imply that the whole nondense set has full Hausdorff dimension, which is a significant result. We are, however, interested in the strengthening to winning because of its stability under intersections. In particular, our result, Theorem 1.1, applies to the smaller set of points with nondense complete orbits because the winning property is stable under intersections and shows that this smaller set also has full Hausdorff dimension. 
Note that the hyperplane absolute winning property is a variant of the winning property and implies it (see Section 2).

Corollary 1.2. Let $\left\{g_{m}\right\}_{m=0}^{\infty}$ be a family of $C^{2}$-Anosov diffeomorphisms preserving $\lambda$ such that $h_{\lambda}\left(g_{m}\right)=h_{\text {top }}\left(g_{m}\right)$ for all $m$. Let $\left\{\boldsymbol{x}_{\ell}\right\}_{\ell=0}^{\infty} \subset \mathbb{T}^{2}$. Then the set

$$
\bigcap_{m=0}^{\infty} \bigcap_{\ell=0}^{\infty}\left(N D\left(g_{m}, \boldsymbol{x}_{\ell}\right) \cap N D\left(g_{m}^{-1}, \boldsymbol{x}_{\ell}\right)\right)
$$

is hyperplane absolute winning (HAW) and thus has full Hausdorff dimension.

REMARK 1.3. Since the countable intersection property holds for all HAW subsets on $\mathbb{T}^{2}$ (see Section 2), we may intersect the set from (1.1) with any countable family of HAW subsets on $\mathbb{T}^{2}$ and still retain the HAW property and thus also retain the property of having full Hausdorff dimension. For example, the intersection of the set from (1.1) with any countable collection of translates of badly approximable vectors on $\mathbb{T}^{2}$ (which is HAW [4, Theorem 2.5]) is still HAW and, thus, has full Hausdorff dimension.

REMARK 1.4. We can also obtain results when the dimension $d$ is greater than two. It follows from the proof of Theorem 1.1 and from (4) of Lemma 2.1 that any Anosov diffeomorphism having a bilipschitz (or quasisymmetric) conjugacy with a hyperbolic automorphism on $\mathbb{T}^{d}$ will have an $\alpha$-winning nondense set for some $\alpha>0$ (see also Section 3.1 ).

Theorem 1.1 answers the question, first raised in [18, Section 6], of whether there are nonlinear dynamical systems with winning nondense sets in dimensions greater than one.

\section{WinNing AND hyPERPLANE ABSOlute WinNing}

The winning property was introduced by W. Schmidt [17 in 1966 and has many later variants (see [4, 10, 16]). We define the winning property and one particular strengthening, the hyperplane absolute winning (HAW) property, for $\mathbb{R}^{d}$. HAW was introduced in [4].

Let $0<\alpha<1$ and $0<\beta<1$. Let $S \subset \mathbb{R}^{d}$ and $\rho(\cdot)$ denote the radius of a closed ball. Two players, Alice $A$ and Bob $B$, alternate choosing nested closed balls

$$
B_{1} \supset A_{1} \supset B_{2} \supset A_{2} \cdots
$$

on $\mathbb{R}^{d}$ according to the following rules:

$$
\rho\left(A_{n}\right)=\alpha \rho\left(B_{n}\right) \quad \text { and } \quad \rho\left(B_{n}\right)=\beta \rho\left(A_{n-1}\right) .
$$

A set $S$ is called $(\alpha, \beta)$-winning if Alice has a strategy guaranteeing $\cap_{i} B_{i}$ lies in $S$ for the given $\alpha$ and $\beta$. A set $S$ is called $\alpha$-winning if it is $(\alpha, \beta)$-winning for the given $\alpha$ and every $\beta$. A set $S$ is called winning if it is $\alpha$-winning for some $\alpha$. These sets have four important properties for us [16, 17]:

Lemma 2.1. Properties of winning sets.

(1) An $\alpha$-winning set in $\mathbb{R}^{n}$ is dense and of full Hausdorff dimension.

(2) A countable intersection of $\alpha$-winning sets is $\alpha$-winning.

(3) An $\alpha$-winning set in $\mathbb{R}^{n}$ with a countable number of points removed is $\alpha$-winning.

(4) The image of an $\alpha$-winning set under a bilipschitz map is $\alpha^{\prime}$-winning, where $\alpha^{\prime}$ depends only on $\alpha$ and the map.

Now fix a $k \in\{0,1, \cdots, d-1\}$ and restrict $0<\beta<1 / 3$. Bob initially chooses $\boldsymbol{x}_{1} \in \mathbb{R}^{d}$ and $r_{1}>0$ and forms a closed ball $B_{1}=B\left(\boldsymbol{x}_{1}, r_{1}\right)$. At each stage of the game, after Bob chooses $\boldsymbol{x}_{i} \in \mathbb{R}^{d}$ and $r_{i}>0$, Alice chooses an affine subspace $L$ of dimension $k$ and removes an $\varepsilon_{i}$-neighborhood $A_{i}=L^{\left(\varepsilon_{i}\right)}$ from $B_{i}:=B\left(\boldsymbol{x}_{i}, r_{i}\right)$ for some $0<\varepsilon_{i} \leq \beta r_{i}$. Then Bob chooses $\boldsymbol{x}_{i+1}$ and $r_{i+1} \geq \beta r_{i}$ such that

$$
B_{i+1}:=B\left(\boldsymbol{x}_{i+1}, r_{i+1}\right) \subset B_{i} \backslash A_{i} .
$$


A set $S$ is said to be $k$-dimensionally $\beta$-absolute winning if Alice has a strategy guaranteeing that $\cap_{i} B_{i}$ intersects $S$. A set $S$ is $k$-dimensionally absolute winning if it is $k$-dimensionally $\beta$-absolute winning for every $0<\beta<1 / 3$. We call $(d-1)$-dimensionally absolute winning sets hyperplane absolute winning $(H A W)$ sets. These sets have three important properties for us [4, Proposition 2.3]:

Lemma 2.2. Properties of $k$-dimensionally absolute winning sets.

(1) $H A W$ (and thus $k$-dimensional absolute winning for all $0 \leq k \leq d-1$ ) implies $\alpha$-winning for all $0<\alpha<1 / 2$.

(2) The countable intersection of $k$-dimensionally absolute winning sets is $k$-dimensionally absolute winning.

(3) The image of a k-dimensionally absolute winning set under a $C^{1}$ diffeomorphism of $\mathbb{R}^{d}$ is $k$-dimensionally absolute winning.

\section{Proofs}

In this section, we prove Theorem 1.1 and its corollary. The two main ingredients are the HAW property, already introduced, and the following smooth classification of Anosov diffeomorphisms of the 2-torus (see [9, Corollary 20.4.5] for example):

Theorem 3.1. Suppose $g: \mathbb{T}^{2} \rightarrow \mathbb{T}^{2}$ is a $C^{2}$-Anosov diffeomorphism preserving $\lambda$ such that $h_{\lambda}(g)=$ $h_{\mathrm{top}}(g)$. Then $g$ is $C^{1}$ conjugate to a linear automorphism.

Proof of Theorem 1.1. The Anosov diffeomorphism $g$ is $C^{1}$-conjugate to a linear automorphism $T$ by Theorem 3.1. Let $h: \mathbb{T}^{2} \rightarrow \mathbb{T}^{2}$ be this conjugacy from the Anosov diffeomorphism to the linear automorphism. The nondense set $N D\left(T, h\left(\boldsymbol{x}_{0}\right)\right)$ is HAW by [4, Theorem 2.6]. Consequently, $N D\left(g, \boldsymbol{x}_{0}\right)$ is HAW by (3) from Lemma 2.2. This proves the desired result.

Proof of Corollary 1.2. The desired result follows from the theorem and (1) and (2) from Lemma 2.2 and (1) from Lemma 2.1.

3.1. Remarks. A key ingredient in the proof of the smooth classification is the fact that the stable and unstable foliations for a $C^{2}$-Anosov diffeomorphism of the 2-torus are $C^{1}$. The proof of the fact relies on a more general result, namely that a codimension-1 stable manifold for a $C^{2}$-Anosov diffeomorphism of a compact manifold forms a $C^{1}$ foliation [ 8 , Corollary 4]. The same applies to the unstable manifold, from which we obtain the desired $C^{1}$ foliations for the 2-torus. This leads to a characterization of smooth conjugacies for the 2-torus [11, 13, 14] (also see [9, Chapter 20.4.b]) and to our smooth classification. (Note that, for a torus of dimension three or greater, either the unstable manifold or the stable manifold will not be codimension-1.)

For dimensions three and greater, we lack a smooth classification theorem. In fact, the characterization of smooth conjugacies is more complicated, and $\mathrm{R}$. de la Llave showed that two Anosov diffeomorphisms of $\mathbb{T}^{d}$ for $d \geq 4$ may be topologically conjugate without being Lipschitz conjugate [12, Theorem 6.3]. (For the 3-torus, the situation is not settled.) A. Gogolev noted that de la Llave's counterexample can be generalized so that (in most cases) one can take one of the diffeomorphisms to be a linear automorphism [7, Theorem B]. Should a smooth classification in higher dimensions be found, it would be more complicated than Theorem 3.1, especially since it must allow for cases in which the conjugacy is only Hölder. On the other hand, we always have a Hölder classification theorem for this context: every Anosov diffeomorphism of $\mathbb{T}^{d}$ is Hölder conjugate to a linear hyperbolic automorphism of $\mathbb{T}^{d}[9$, Theorems 18.6.1 and 19.1.2]. This Hölder classification is, however, not strong enough for us because we need the conjugacy to be quasisymmetric or bilipschitz (see [16] and (4) from Lemma 2.1) to carry out the proof in this paper.

Another way of approaching this problem for dimensions three and greater that should avoid using a smooth classification is to employ a technique used earlier [18, 21, namely using a bounded distortion property (i.e. universal bounds on the ratio of the absolute value of the Jacobian determinant for nearby points - see [18, Section 2.1.1] for example). If we assume conformality, bounded 
distortion follows. However, without this assumption (in dimensions two or greater), bounded distortion may not follow, and without it we may not be able to control the local "shape" of successive iterates of small balls. Since the variants of the game involve intersections with balls, this lack of control is an obstacle that needs to be overcome if this approach were to succeed for more general nonlinear maps.

Also, we note that for linear maps, one can replace bounded distortion with linear properties of the map. In particular, one can decompose $\mathbb{R}^{d}$ into a direct sum of subspaces appropriate for the linear action and, using the HAW property, one is allowed to remove "bad" affine hyperplanes [4]. Linearity seems important to this step and may not possible with a nonlinear system.

Finally, we mention a theorem closely related to our results. For pairs of toral mappings, one can consider the set of points with orbits that are nondense under one of the pair and dense under the other, a problem first considered in [2]. The nonlinear case for this setup of simultaneous dense and nondense orbits is also interesting, and, for generic pairs of $C^{2}$-Anosov diffeomorphisms of $\mathbb{T}^{2}$, it is known that the desired set is uncountable and dense [19, Theorem 1.3].

\section{REFERENCES}

[1] A. G. Abercrombie and R. Nair, An exceptional set in the ergodic theory of expanding maps on manifolds, Monatsh. Math. 148 (2006), 1-17.

[2] V. Bergelson, M. Einsiedler, and J. Tseng, Simultaneous dense and nondense orbits for commuting maps, Israel J. Math. 210 (2015), 23-45.

[3] R. Broderick, L. Fishman, and D. Kleinbock, Schmidt's game, fractals, and orbits of toral endomorphisms, Ergodic Theory Dynam. Systems 31 (2011), 1095-1107.

[4] R. Broderick, L. Fishman, D. Kleinbock, A. Reich, and B. Weiss, The set of badly approximable vectors is strongly $C^{1}$ incompressible. Math. Proc. Cambridge Philos. Soc. 153 (2012), no. 2, 319-339.

[5] S. G. Dani, On orbits of endomorphisms of tori and the Schmidt game, Ergodic Theory Dynam. Systems 8 (1988), 523-529.

[6] D. Färm, Simultaneously non-dense orbits under different expanding maps. Dyn. Syst. 25 (2010), 531-545.

[7] A. Gogolev, Smooth conjugacy of Anosov diffeomorphisms on higher-dimensional tori, J. Mod. Dyn. 2 (2008), no. 4, 645-700.

[8] M. W. Hirsch and C. C. Pugh, Stable manifolds and hyperbolic sets. 1970 Global Analysis (Proc. Sympos. Pure Math., Vol. XIV, Berkeley, Calif., 1968) pp. 133-163 Amer. Math. Soc., Providence, R.I.

[9] A. Katok and B. Hasselblatt, "Introduction to the modern theory of dynamical systems. With a supplementary chapter by Katok and Leonardo Mendoza." Encyclopedia of Mathematics and its Applications, 54. Cambridge University Press, Cambridge, 1995.

[10] D. Kleinbock and B. Weiss, Modified Schmidt games and Diophantine approximation with weights, Adv. Math. 223 (2010), 1276-1298.

[11] R. de la Llave, Invariants for smooth conjugacy of hyperbolic dynamical systems. II. Comm. Math. Phys. 109 (1987), no. 3, 369-378.

[12] R. de la Llave, Smooth conjugacy and $S$-R-B measures for uniformly and non-uniformly hyperbolic systems, Comm. Math. Phys. 150 (1992), no. 2, 289-320.

[13] J. M. Marco and R. Moriyn, Invariants for smooth conjugacy of hyperbolic dynamical systems. I. Comm. Math. Phys. 109 (1987), no. 4, 681-689.

[14] J. M. Marco and R. Moriyn, Invariants for smooth conjugacy of hyperbolic dynamical systems. III. Comm. Math. Phys. 112 (1987), no. 2, 317-333.

[15] B. Mance and J. Tseng, Bounded Lüroth expansions: applying Schmidt games where infinite distortion exists, Acta Arith. 158 (2013), 33-47.

[16] C. McMullen, Winning sets, quasiconformal maps and Diophantine approximation, Geom. Funct. Anal. 20 2010, 726-740.

[17] W. Schmidt, Badly approximable numbers and certain games, Trans. Amer. Math. Soc. 123 (1966), 178-199.

[18] J. Tseng, Schmidt games and Markov partitions, Nonlinearity 22 (2009), 525-543.

[19] J. Tseng, Simultaneous dense and nondense orbits for toral diffeomorphisms, Ergodic Theory and Dynamical Systems, to appear. http://arxiv.org/abs/1406.1970

[20] M. Urbański, The Hausdorff dimension of the set of points with nondense orbit under a hyperbolic dynamical system, Nonlinearity 4 (1991), 385-397.

[21] W. Wu, Schmidt games and non-dense forward orbits of certain partially hyperbolic systems, Ergodic Theory and Dynamical Systems, to appear. http://dx.doi.org/10.1017/etds.2014.136 
School of Mathematics, University of Bristol, University Walk, Bristol, BS8 1TW UK

E-mail address: j.tseng@bristol.ac.uk jimmytseng01@gmail.com 